\title{
Inquéritos domiciliares nacionais de base populacional em saúde: uma revisão narrativa
}

\author{
Nationwide population-based household surveys in health: \\ a narrative review
}

Vinicius Siqueira Tavares Meira Silva (https://orcid.org/0000-0002-3271-0811) ${ }^{1}$

Luiz Felipe Pinto (http://orcid.org/0000-0002-9888-606X) ${ }^{2}$

${ }^{1}$ Programa de Residência em Medicina de Família e Comunidade, Secretaria Municipal de Saúde do Rio de Janeiro. R. Evaristo da Veiga 16, Centro. 20031-040 Rio de Janeiro RJ Brasil.

vinicius.smeira@gmail.com

${ }^{2}$ Departamento de Medicina em Atenção Primária

à Saúde, Faculdade de

Medicina, Universidade Federal do Rio de Janeiro. Rio de Janeiro RJ Brasil.

\begin{abstract}
Household surveys are one of the primary methodologies used in population-based studies. This narrative review of the literature aims to gather and describe the leading national and international household surveys of relevance. In Brazil, the historical role played by the Brazilian Institute of Geography and Statistics (IBGE) in conducting the most relevant research in the production of social data stands out. The Medical-Health Care Survey (AMS) and the National Household Sample Survey (PNAD), with the serial publication of Health Supplements, are the country's primary sources of health information. In 2013, in partnership with the Ministry of Health, IBGE launched the National Health Survey (PNS), the most significant household health survey ever conducted in Brazil. The PNS-2019 received a major thematic and sampling expansion and, for the first time, applied the Primary Care Assessment Tool to assess PHC services in all 27 Brazilian states.
\end{abstract}

Key words Household survey, Sampling, PCAT, Brazil
Resumo Os inquéritos domiciliares representam uma das principais metodologias empregadas em estudos de base populacional. O objetivo desta revisão narrativa da literatura é reunir e descrever os principais inquéritos domiciliares em saúde de relevância nacional e internacional. No Brasil, destaca-se o papel histórico desempenhado pelo Instituto Brasileiro de Geografia e Estatística (IBGE) na condução das pesquisas mais importantes na produção de dados sociais. A Pesquisa de Assistência Médico-Sanitária (AMS) e a Pesquisa Nacional por Amostra de Domicílios (PNAD), com a publicação episódica de Suplementos Saúde, consistem das principais fontes de informação em saúde no país. Em 2013, o IBGE, em convênio com o Ministério da Saúde, lançou a Pesquisa Nacional de Saúde (PNS), o maior inquérito domiciliar em saúde já realizado no Brasil. A PNS-2019 recebeu uma grande ampliação, tanto temática, quanto ao tamanho de sua amostra, e, pela primeira vez, aplicou o instrumento Primary Care Assessment Tool para avaliação dos serviços de Atenção Primária em Saúde em todas as unidades da federação do Brasil.

Palavras-chave Inquéritos domiciliares, Amostragem, PCAT, Brasil 


\section{Introdução}

A produção de informação em saúde é indispensável para orientar o planejamento, a organização, o monitoramento, a avaliação e a qualificação de qualquer serviço ou política de saúde. Existem inúmeras ferramentas e métodos que podem ser aplicados para se obter uma informação em saúde. Aquele capaz de fornecer ao gestor e à sociedade a informação desejada é a escolha mais adequada.

O inquérito populacional é uma metodologia aplicada com a finalidade de produzir informações em saúde, as quais são inferidas a partir das respostas obtidas em entrevista aplicada a uma amostra probabilística significativa da população analisada. Em geral, são estudos transversais, embora também possam ser longitudinais (ou ainda, painéis repetidos), que estão circunscritos a uma única área ou a algumas regiões, podendo ou não ter um segmento demográfico específico como alvo. A coleta de dados é feita de maneira sistemática a partir de um questionário administrado na casa da pessoa por um entrevistador treinado. $\mathrm{O}$ questionário pode ser original ou mesmo integrar algum instrumento validado estatisticamente. Trata-se de uma ferramenta importante, sobretudo em países em desenvolvimento, que ainda dispõem de sistemas de informação incipientes para fornecer dados confiáveis e abrangentes em saúde ${ }^{1}$.

Podendo abranger uma grande variedade de temas ou se restringir a poucos assuntos, os inquéritos têm uma singularidade em relação a muitos métodos, pois abordam o indivíduo em seu domicílio, ao invés de realizar a entrevista nas unidades de saúde ${ }^{2}$. Na entrevista, obtém-se dados sobre características sociodemográficas e da saúde da população, como estado de saúde, hábitos, estilo de vida, utilização e consumo de serviços etc. Eventualmente, são pesquisados valores de parâmetros físicos (peso, altura, pressão arterial) e laboratoriais, pela coleta de espécimes clínicos, como sangue e urina. Os inquéritos probabilísticos também estimam os denominadores populacionais que são a chave para o cálculo de indicadores de interesse para o escopo dos estudos.

$\mathrm{Na}$ definição da amostra em inquéritos de base populacional, frequentemente é utilizada a amostragem por conglomerados em múltiplos estágios ("amostragem de áreas"), sendo a unidade amostral um agrupamento populacional definido - como um setor censitário - que a cada etapa é decomposto em sub-grupos, até alcançar o objeto de interesse do estudo ${ }^{3}$.
Esta revisão narrativa apresentará os principais inquéritos populacionais em saúde do mundo e do Brasil, desde aqueles com relevância histórica até pesquisas mais recentes. Além disso, serão detalhados e analisados os principais inquéritos populacionais brasileiros, fundamentais na produção de informação em saúde no país, como algumas edições especiais da Pesquisa $\mathrm{Na}$ cional por Amostra de Domicílios (PNAD) e a Pesquisa Nacional de Saúde (PNS).

\section{Inquéritos domiciliares nacionais de base populacional no mundo}

Desde a década de 1990, a Organização Mundial da Saúde (OMS), na tentativa de incentivar a produção de informação para orientar a política de saúde, sobretudo entre os países em desenvolvimento, e a fim de uniformizar os indicadores entre os distintos países, de modo a torná-los comparáveis, orientou a produção de dados populacionais, como de morbidade, necessidades especiais, utilização do serviço de saúde, estilo de vida, aleitamento materno, peso ao nascer e outros. Além de designar os indicadores, a entidade recomendou a realização do inquérito domiciliar para recolher os dados.

Bem antes disso, contudo, os Estados Unidos da América realizaram o seu primeiro inquérito nacional em saúde em 1935-1936. E a seguir, O National Health Interview Survey (NHIS) passou a ser realizado desde a metade da década de 1950, seguindo até os dias de hoje. Nele são coletados dados socioeconômicos, demográficos e de saúde dos norte-americanos. Hoje em dia, uma série de outros inquéritos populacionais com escopos mais específicos é desenvolvida nesse país. Também já na década de 1970, o Reino Unido começou a aplicar um inquérito nacional de caráter longitudinal, o General Health Survey (GHS), que foi seguido de outros estudos semelhantes, como no Canadá que desde a década de 2000 leva a campo anualmente a Canadian Community Health Survey (CCHS) $)^{5,6}$.

A partir de 2006, a União Europeia (UE) passou a conduzir um grande inquérito domiciliar entre seus países-membros, o chamado European Health Interview Survey (EHIS), que tem sido aplicado para indivíduos acima dos 15 anos. Em sua primeira versão (EHIS 1), a realização do inquérito era facultativa e participaram 17 países europeus, entre 2006 e 2009. Com uma periodicidade quinquenal, a partir da sua segunda edição (EHIS 2), a pesquisa passou a ser obrigatória para todos os países-membros, conforme decisão 
do Parlamento Europeu. A EHIS-2 foi aplicada em todos os signatários do bloco, mais a Noruega e a Islândia, entre 2013 e 2015, adotando um questionário único, assim como metodologias de amostragem e aplicação, de modo a gerar dados harmônicos com alto grau de comparação. A pesquisa foi dividida em quatro módulos: (1) estado de saúde, (2) serviços de saúde, (3) determinantes de saúde e (4) variáveis demográficas e socioeconômicas. Sua terceira versão, o EHIS-3, foi planejada para ir a campo em $2019^{7}$.

Alguns países europeus também desenvolvem relevantes pesquisas domiciliares. Em Portugal, o Inquérito Nacional de Saúde (INS) é realizado pelo Instituto Nacional de Estatística (INE) e cobre todo o território nacional. Além de recolher os dados sociodemográficos dos indivíduos entrevistados, o questionário traz perguntas sobre o estado de saúde, os cuidados em saúde e os determinantes da saúde ${ }^{8}$. Uma pesquisa semelhante tem sido realizada na Espanha, a Encuesta Nacional de Salud (ENS), com periodicidade quinquenal, apresentou seus últimos resultados em $2017^{9}$. A Itália é outro país pioneiro no desenvolvimento de inquéritos domiciliares em saúde, a Indagini Multiscopo sulle Famiglie (IMSF), realizada anualmente desde $1993^{10}$.

A China, país mais populoso do mundo, realizou seu primeiro inquérito domiciliar de saúde envolvendo o período de 2012 a 2017 . A pesquisa nacional atingiu 11 províncias das 23 existentes, e dez grupos étnicos, o que corresponde a $40 \%$ da população minoritária no país. O questionário abrangeu os seguintes temas: características socioeconômicas e demográficas, estilo de vida, morbidade pessoal e familiar. Houve também mensuração de parâmetros fisiológicos através de análises laboratoriais, avaliação antropométrica, exame físico e estudos complementares, como prova de função pulmonar e medida da densidade mineral óssea. As amostras de sangue obtidas passaram a compor um banco multiétnico para estudos genéticos futuros ${ }^{11}$.

Outro país asiático de dimensões parecidas, a Índia, também operou um grande estudo populacional, o National Family Health Survey (NFHS). Em sua quarta edição, o NFHS (20152016) alcançou todas as províncias do país e integrou questões socioeconômicas, demográficas e de habitação e assuntos relacionados à saúde, como morbidades, violência, atenção à saúde da mulher e da criança, comportamento sexual, planejamento familiar, estilo de vida, nutrição, utilização de serviços de saúde e cobertura de seguros. Ainda foram obtidos dados de avalia- ção antropométrica, aferição da pressão arterial e análises laboratoriais ${ }^{12}$.

Na Oceania, o sistema nacional de saúde da Austrália conduz inquéritos domiciliares há algumas décadas. A última versão da National Health Survey (NHS), realizada entre 2017 e 2018, trouxe uma diversidade de dados sobre a composição da população em todo o país, assim como informações sobre rede de saúde, estado de saúde, morbidades crônicas, hábitos e estilo de vida, avaliação antropométrica e valores da pressão arterial na entrevista ${ }^{13}$. Seu país vizinho, a Nova Zelândia, desde 2011, realiza uma pesquisa populacional contínua em saúde, a New Zealand Health Survey (NZHS), que verifica indicadores de saúde, hábitos, estilo de vida e acesso ao sistema de saúde, tanto para adultos como para crianças em todo o país. Também já realizou a (SoFIE-Primary Care) que utilizou algumas perguntas de avaliação da atenção primária à saúde, a partir do instrumento do Primary Care Assessment Tool $(\text { PCAT })^{14}$.

\section{Inquéritos domiciliares nacionais de base populacional no Brasil}

O Instituto Brasileiro de Geografia e Estatística (IBGE) é o órgão oficial de Estatística e Geografia do Brasil e o principal responsável pela realização de inquéritos nacionais domiciliares desde os primórdios do primeiro Censo Demográfico, em 1872. Historicamente, a Pesquisa Nacional por Amostra de Domicílios (PNAD), criada na década de 1960 para atualizar os dados populacionais em períodos intercensitários, e a Pesquisa de Assistência Médico-Sanitária (AMS), criada na década de 1970, são considerados os dois maiores inquéritos brasileiros. Ambos continuam gerando informações administrativas e apresentando resultados relevantes para a saúde.

\section{Pesquisa de Assistência Médico-Sanitária (AMS)}

O primeiro inquérito nacional de cunho administrativo do Brasil foi oficialmente lançado no ano de 1975 a partir de um convênio do IBGE com o Ministério da Saúde. A Pesquisa de Assistência Médico-Sanitária (AMS) permitiu a atualização dos dados administrativos de estabelecimentos de saúde. A AMS foi realizada anualmente por um longo período, o qual se encerrou em $2009^{15}$.

Seu objetivo tem sido o de caracterizar o perfil da capacidade instalada de serviços de saúde 
no Brasil, incluindo em seu universo todos os estabelecimentos que prestam assistência em saúde do país, sejam públicos ou privados, unidades com regime ambulatorial ou oferta de internação, assim como serviços de diagnose, terapia e controle de zoonoses. Além de contabilizar os serviços existentes em todo o território nacional, a AMS fornece informações adicionais relacionadas às modalidades de serviços prestados, instalações, recursos humanos, leitos e internações, equipamentos e tecnologias, entre outras.

Contudo, já em 2002, o Ministério da Saúde elaborou o Cadastro Nacional de Estabelecimentos de Saúde (CNES) e passou, gradualmente, a deixar de apoiar o IBGE na elaboração da AMS, o que representa uma enorme perda para o inquérito administrativo quase censitário que registrava periodicamente informações únicas. Sua última publicação, em 2009, inovou ao coletar os dados através de questionário eletrônico diretamente respondido pelo informante via web. Nesta edição, também foi registrada a localização geográfica de cada estabelecimento, utilizando o global positioning system (GPS), o que permitiu grande precisão na análise da distribuição espacial dos serviços pelo território brasileiro.

\section{Pesquisa Nacional por Amostra de Domicílios (PNAD)}

No Brasil, as primeiras informações de saúde oriundas de um inquérito domiciliar vieram da Pesquisa Nacional por Amostra de Domicílios (PNAD), que foi implementada em 1967 com o propósito de investigar aspectos da população e do domicílio. A PNAD é planejada e executada pelo IBGE até os dias de hoje e gradativamente foi ampliando a sua abrangência até alcançar, em 2004, representatividade nacional ${ }^{16}$.

A pesquisa teve uma grande variação na sua periodicidade desde que foi implementada, até que, em 2012, tornou-se contínua em todo o território nacional. Com a criação do Sistema Integrado de Pesquisas Domiciliares (SIPD), tendo como um dos pilares a PNAD Contínua (PNAD-C), o IBGE tem buscado coordenar o planejamento e execução de diversos outros inquéritos domiciliares realizados pelo Instituto, a fim de ampliar sua abrangência e otimizar a utilização de recursos. O SIPD reúne, atualmente, a PNAD-C, a Pesquisa Nacional de Saúde (PNS) e a Pesquisa de Orçamentos Familiares (POF). Neste contexto, estabeleceu uma "amostra mestra" que atende a todos as pesquisas do SIPD, apoiando-se no Censo Demográfico de 2010 para selecionar as unidades de área que constróem as subamostras de cada estudo ${ }^{17}$.

A cada trimestre, são visitados em torno de 200 mil domicílios e coletadas informações sobre trabalho, incluindo eventualmente trabalho infantil, voluntário e doméstico, além de características demográficas, de educação e da habitação, incluindo as condições de moradia (ocupação, abastecimento de água, rede de esgoto, existência de banheiro, destino do lixo, iluminação elétrica) e a presença de bens de consumo (telefone celular e fixo, automóvel, motocicleta, televisão, microcomputador, conexão à Internet, entre outros) ${ }^{18}$.

Durante esses anos, a PNAD realizou abordagens específicas para algumas áreas temáticas em complementação ao seu questionário básico. Em 1981 e 1986, foram investigados aspectos relacionados à morbidade referida, utilização dos serviços de saúde, suplementação alimentar e anticoncepção. Pesquisadores apontam algumas limitações do inquérito de 1981, em que não houve independência nas questões sobre morbidade e procura pelo serviço de saúde e também houve a pré-fixação de uma data nas questões sobre os mesmos temas (p. ex. "No período de 1 a 14 de novembro de $1981 . .$. "), o que prejudicou a qualidade das respostas na medida em que a entrevista foi aplicada em diferentes tempos de recordação. Apenas o último problema foi corrigido na versão de 1986, relacionando o tempo de referência com o momento da entrevista (p. ex. "Nas duas últimas semanas...”) ${ }^{19}$.

Financiado pelo Ministério da Saúde (MS), o suplemento de 1998 interrompeu um período de dez anos sem informações de base populacional em saúde, no Brasil. Foi o primeiro de uma série quinquenal de suplementos sobre saúde na PNAD (1998, 2003, 2008). Desde 1998, o questionário passou por uma série de adaptações e foi gradativamente ampliado na busca de diversificar as características de saúde dos moradores pesquisadas (Quadro 1).

O suplemento Saúde da PNAD- $2003^{20}$ manteve basicamente a mesma estrutura do questionário de 1998. As principais alterações foram a retirada do tópico sobre gastos privados em saúde e a inclusão de questões relativas ao acesso a serviços preventivos de saúde para mulher a partir dos 25 anos de idade, como exame clínico das mamas, mamografia e exame preventivo do câncer de colo do útero. Em relação à investigação da prevalência de doenças crônicas na população, também houve uma alteração na formulação das questões, que passaram a requerer que houvesse um diagnóstico fornecido por um profissional 
Quadro 1. Características de saúde dos moradores pesquisadas simultaneamente nos Suplementos de Saúde da PNAD/IBGE de 1998, 2003 e 2008.

\begin{tabular}{|c|c|c|}
\hline PNAD-1998 & PNAD-2003 & PNAD-2008 \\
\hline $\begin{array}{c}\mathrm{N}^{\circ} \text { de módulos: } 7 \\
\mathrm{~N}^{\circ} \text { de perguntas: } 84\end{array}$ & $\begin{array}{c}\mathrm{N}^{\circ} \text { de módulos: } 7 \\
\mathrm{~N}^{\circ} \text { de perguntas: } 85\end{array}$ & $\begin{array}{c}\mathrm{N}^{\circ} \text { de módulos: } 13 \\
\mathrm{~N}^{\circ} \text { de perguntas: } 242\end{array}$ \\
\hline \multicolumn{3}{|c|}{ Morbidade } \\
\hline $\begin{array}{l}\text { Auto-avaliação do estado de saúde a } \\
\text { partir de uma escala de cinco graus } \\
\text { (de "muito ruim" a "muito bom") e do } \\
\text { impacto sobre as atividades habituais; } \\
\text { presença percebida ou diagnosticada de } \\
\text { alguma das doenças crônicas listadas } \\
\text { (doença de coluna ou das costas, } \\
\text { artrite ou reumatismo, câncer, diabetes, } \\
\text { bronquite ou asma, hipertensão ou } \\
\text { pressão alta, doença do coração, doença } \\
\text { renal crônica, depressão, tuberculose, } \\
\text { tendinite ou tenossinovite e cirrose), } \\
\text { selecionadas com base em três critérios } \\
\text { (alta prevalência, possibilidade de } \\
\text { intervenção e confiabilidade observada } \\
\text { em outros estudos). }\end{array}$ & $\begin{array}{l}\text { A maior parte do questionário foi } \\
\text { mantida; a alteração mais notória } \\
\text { está nas questões sobre a presença } \\
\text { dos problemas crônicos listados; } \\
\text { enquanto na versão de 1998, a } \\
\text { indagação era aberta (“... tem } \\
\text { hipertensão?”), em 2003, passou- } \\
\text { se a questionar a existência do } \\
\text { diagnóstico a partir da alegação } \\
\text { de um “médico ou profissional de } \\
\text { saúde”, dispensando a percepção da } \\
\text { pessoa. }\end{array}$ & $\begin{array}{l}\text { Não houve alterações em } \\
\text { relação ao questionário } \\
\text { anterior. }\end{array}$ \\
\hline \multicolumn{3}{|c|}{ Cobertura de planos de saúde } \\
\hline $\begin{array}{l}\text { Frequência de contratação dos planos } \\
\text { de saúde (médicos e odontológicos); } \\
\text { natureza particular, empresarial } \\
\text { ou instituição pública; avaliação } \\
\text { do plano em escala; características } \\
\text { socioeconômicas e demográficas das } \\
\text { pessoas cobertas por planos; perfil dos } \\
\text { titulares, dependentes e agregados; } \\
\text { serviços cobertos pelos planos; despesas } \\
\text { efetuadas com as mensalidades dos } \\
\text { planos. }\end{array}$ & $\begin{array}{l}\text { O questionário anterior é } \\
\text { mantido, com o acréscimo de } \\
\text { apenas uma pergunta sobre o } \\
\text { tipo de acomodação (quarto ou } \\
\text { apartamento privativo, coletivo ou } \\
\text { enfermaria) oferecida pelo plano. }\end{array}$ & $\begin{array}{l}\text { O questionário anterior é } \\
\text { mantido na maior parte, com } \\
\text { acréscimo de uma questão } \\
\text { sobre o tempo sem interrupção } \\
\text { a que o entrevistado tem } \\
\text { direito ao plano e outra sobre a } \\
\text { quantidade de planos de saúde } \\
\text { o entrevistado tem direito. }\end{array}$ \\
\hline
\end{tabular}

continua

de saúde para a resposta positiva (p. ex. "Algum médico ou profissional de saúde já disse que ... tem diabetes?), enquanto, em 1998, a percepção da pessoa bastava (p. ex. “... tem diabetes”).

Já em 2008, o Suplemento Saúde da PNAD ${ }^{21}$ triplicou de tamanho em número de perguntas, recebendo mais algumas questões relacionadas aos cuidados de saúde das mulheres a partir dos 25 anos, com a caracterização do acesso aos exames preventivos a partir da indagação sobre pagamento, cobertura por plano de saúde ou realização no SUS, e mais um questionamento sobre histerectomia. Incluiu-se a investigação de fatores de risco e proteção à saúde com questões relacionadas à realização de atividade física, atividades de lazer, utilização de TV, computador e videogames, consumo de tabaco, acidentes de trânsito, uso de cinto de segurança e vítimas de algum tipo de violência e seu impacto nas atividades habituais.

Na PNAD 2008, foram investigados os domicílios registrados no, então, Programa Saúde da Família (PSF), o momento da realização desse registro e a frequência de visitação do agente comunitário de saúde ou de algum profissional de saúde. No país, 47,7\% dos domicílios entrevistados declararam estar filiados ao PSF, com um percentual maior na região Nordeste $(64,8 \%)$, seguida das regiões Norte (51,0\%), Sul (50,3\%), Centro-Oeste $(49,1 \%)$ e Sudeste $(35,9 \%)$.

\section{De suplemento especial da PNAD à PNS-2013: vida própria}

A partir de 2013, com o apoio do Ministério da Saúde, o Suplemento Saúde da PNAD-2008 
Quadro 1. Características de saúde dos moradores pesquisadas simultaneamente nos Suplementos de Saúde da PNAD/IBGE de 1998, 2003 e 2008.

\begin{tabular}{|c|c|c|}
\hline PNAD-1998 & PNAD-2003 & PNAD-2008 \\
\hline $\begin{array}{c}N^{\circ} \text { de módulos: } 7 \\
N^{\circ} \text { de perguntas: } 84\end{array}$ & $\begin{array}{c}\mathrm{N}^{\circ} \text { de módulos: } 7 \\
\mathrm{~N}^{\circ} \text { de perguntas: } 85\end{array}$ & $\begin{array}{c}\mathrm{N}^{\circ} \text { de módulos: } 13 \\
\mathrm{~N}^{\circ} \text { de perguntas: } 242\end{array}$ \\
\hline \multicolumn{3}{|c|}{ Acesso a serviços de saúde } \\
\hline $\begin{array}{l}\text { Verificar o hábito de recorrer a } \\
\text { um mesmo prestador de saúde } \\
\text { e caracterizá-lo (formais, como } \\
\text { farmácia, hospital, posto, ambulatório, } \\
\text { profissional de saúde etc., ou informais, } \\
\text { como centro espírita, curandeiro etc.); } \\
\text { frequência de consultas médicas e } \\
\text { odontológicas }\end{array}$ & $\begin{array}{l}\text { Não houve alterações em relação ao } \\
\text { questionário anterior. }\end{array}$ & $\begin{array}{l}\text { São adicionadas perguntas sobre } \\
\text { medicamentos de uso contínuo; } \\
\text { disponibilidade da dispensação } \\
\text { gratuita ou custeio próprio } \\
\text { destes; às questões sobre a } \\
\text { frequentação do dentista, foram } \\
\text { agregadas outras quanto à fonte } \\
\text { do serviço (plano, privado ou } \\
\text { SUS). }\end{array}$ \\
\hline \multicolumn{3}{|c|}{ Utilização dos serviços de saúde } \\
\hline $\begin{array}{l}\text { Captar a procura por um atendimento } \\
\text { relacionado à saúde, o motivo e a } \\
\text { frequência; o tipo de prestador do } \\
\text { serviço; a consumação ou não do } \\
\text { atendimento; serviço público ou } \\
\text { privado; prescrição e dispensação } \\
\text { de medicamento no atendimento; } \\
\text { cobrança e fonte do pagamento pelo } \\
\text { atendimento; o nível de satisfação com } \\
\text { o atendimento recebido; motivos de } \\
\text { não procura.. }\end{array}$ & $\begin{array}{l}\text { Não houve alterações significativas } \\
\text { em relação ao questionário anterior. }\end{array}$ & $\begin{array}{l}\text { As perguntas acerca da } \\
\text { prescrição, e acesso a } \\
\text { medicamentos foram } \\
\text { ampliada; foi descartada o } \\
\text { questionamento sobre o nível } \\
\text { de satisfação do usuário com o } \\
\text { serviço. }\end{array}$ \\
\hline \multicolumn{3}{|c|}{ Internação } \\
\hline $\begin{array}{l}\text { No período de referência de } 12 \\
\text { meses, captar as pessoas que tiveram } \\
\text { internação e caracterizar quanto à } \\
\text { frequência, duração, motivo, tipo } \\
\text { de atendimento recebido; se serviço } \\
\text { público ou privado e fonte pagadora; } \\
\text { avaliação do atendimento }\end{array}$ & $\begin{array}{l}\text { Não houve alterações em relação ao } \\
\text { questionário anterior. }\end{array}$ & $\begin{array}{l}\text { Não houve alterações em } \\
\text { relação ao questionário } \\
\text { anterior. }\end{array}$ \\
\hline \multicolumn{3}{|c|}{ Mobilidade física $>14$ anos } \\
\hline $\begin{array}{l}\text { Mensurar, com base em uma escala } \\
\text { progressiva, o grau de dificuldade } \\
\text { com que a pessoa exerce atividades } \\
\text { cotidianas, possibilitando conhecer o seu } \\
\text { estágio de limitação física. (IBGE, 1998) }\end{array}$ & $\begin{array}{l}\text { Não houve alterações em relação ao } \\
\text { questionário anterior. }\end{array}$ & $\begin{array}{l}\text { O módulo foi ampliado, } \\
\text { incluindo perguntas sobre a } \\
\text { prática de atividade física }\end{array}$ \\
\hline Gastos com saúde & \multicolumn{2}{|c|}{ Acesso a serviços de prevenção em mulheres $>25$ anos } \\
\hline $\begin{array}{l}\text { Estimar o total de gastos com bens e } \\
\text { serviços de saúde: mensalidades do } \\
\text { plano de saúde, consultas médicas e de } \\
\text { outros profissionais, hospitalizações, } \\
\text { enfermagem domiciliar, exames, } \\
\text { tratamento dentário, óculos, artigos } \\
\text { ortopédicos e outros. Este módulo foi } \\
\text { excluído nas edições subsequentes. }\end{array}$ & $\begin{array}{l}\text { Tempo decorrido desde o último } \\
\text { exame clínico das mamas, } \\
\text { mamografia e exame preventivo do } \\
\text { câncer de colo do útero }\end{array}$ & $\begin{array}{l}\text { Foram adicionadas questões } \\
\text { sobre o a fonte de pagamento } \\
\text { dos serviços (próprio bolso, } \\
\text { plano de saúde, SUS) e sobre a } \\
\text { realização de histerectomia. }\end{array}$ \\
\hline
\end{tabular}

Nota: A PNAD-2008 incorporou novos módulos não presentes nas edições de 1998 e 2003. Foram eles: atendimento de urgência no domicílio, violência, acidente de trânsito, sedentarismo, tabagismo para a população em geral e uma pesquisa especial de tabagismo para pessoas com 15 anos ou mais.

Fonte: Elaboração dos autores.

foi desvinculado dessa pesquisa nacional e deu origem à Pesquisa Nacional de Saúde (PNS), que logo passou a compor o SPID, junto de outros inquéritos domiciliares do IBGE. Dessa maneira, 
desde a sua primeira versão, a PNS se valeu da "amostra mestra" da PNAD para definir as suas unidades primárias de amostragem (UPA), o que garante maior alcance e representatividade estatística e geográfica em todo o território nacional.

A PNS-2013 contava com 31 módulos e 751 perguntas. Visitou 81.167 domicílios, realizando 64.348 entrevistas com todos os moradores de um mesmo domicílio e 60.202 entrevistas com os moradores selecionados a partir do $12^{\circ}$ módulo. Além disso, foi pioneira no Instituto ao introduzir a coleta de espécimes clínicos dos entrevistados, como amostras de sangue e urina, e submetê-los a análises laboratoriais. Foram indicados para a coleta de material biológico $25 \%$ dos setores censitários pesquisados ${ }^{22,23}$.

Desde sua origem, a PNS vem adotando abordagem similar a dos principais inquéritos de saúde nacionais e internacionais. Isto permite, por exemplo, a comparação dos seus dados com os da série de Suplementos Saúde das PNAD e da Pesquisa de Vigilância de Fatores de Risco e Proteção para Doenças Crônicas por Inquérito Telefônico (VIGITEL) da SVS/Ministério da Saúde. Além disso, passou a incluir temas relevantes para a saúde pública e de interesse do Ministério da Saúde ${ }^{24}$.

O questionário da PNS-2013 ${ }^{25}$ ficou dividido em três partes: (1) módulo domiciliar: com questões para a caracterização do domicílio, incluindo a presença de animais domésticos, e sobre visitas domiciliares de equipe da Estratégia Saúde da Família (ESF) e de agente de endemias; (2) módulo sobre características de todos os moradores do domicílio, que visava a analisar aspectos gerais dos indivíduos, como informações sobre educação, trabalho, rendimento, escolaridade dos menores, portadores de necessidades especiais, cobertura de plano de saúde, utilização de serviços de saúde, condição de saúde dos maiores de 60 anos, cobertura de mamografia; e (3) módulo com morador selecionado, que contemplava perguntas adicionais sobre características do trabalho e apoio social, percepção do estado de saúde, acidentes e violência, estilo de vida, doenças crônicas, saúde da mulher, acompanhamento pré-natal, saúde bucal e atendimento médico. Os dois primeiros módulos podiam ser respondidos por qualquer morador apto a fornecer esses dados e a terceira pelo adulto com 18 anos ou mais aleatoriamente selecionado.

Foram coletadas medidas antropométricas dos moradores selecionado, como peso, altura e circunferência da cintura e realizada aferição da pressão arterial. Os entrevistadores foram previa- mente treinados para realizar estas tarefas. Parte dos indivíduos ainda foram submetidos à coleta de sangue e urina em laboratório indicado, em cujas amostras foram analisados: hemoglobina glicada, colesterol total, LDL, HDL, hemograma, hemoglobina $S$ e outras, creatinina e sorologia para dengue, bem como dosagens urinárias de sódio, potássio e creatinina. Essas amostras foram armazenadas e passaram a compor uma soroteca da população brasileira ${ }^{26}$.

\section{Atenção Primária à Saúde na PNS-2013}

No Brasil, a PNS-2013 foi o primeiro inquérito com amostragem probabilística de abrangência nacional, regional e local a verificar o alcance dos serviços de Atenção Primária à Saúde (APS) por intermédio das equipes de Saúde da Família (eSF), mensurando a porcentagem de domicílios registrados. No total, estimou-se que, em 2013, 34,8 milhões de domicílios estavam vinculados a uma eSF, perfazendo $53,4 \%$ do total de domicílios brasileiros. A proporção foi maior no Nordeste $(64,7 \%)$ e menor na região Sudeste $(46,0 \%)$. A parcela de domicílios registrados nas áreas urbanas $(50,6 \%)$ foi inferior do que nas zonas rurais (70,9\%); a mesma relação se manteve quando questionados sobre se recebiam visitas mensais de algum membro da equipe de eSF (62,7\% entre os domicílios rurais $\mathrm{x} 43,6 \%$ nas zonas urbanas). Este mesmo índice foi menor no Sudeste $(41,6 \%)$ e maior no Centro-Oeste $(58,3 \%)^{27}$.

\section{Pesquisa Nacional de Saúde 2019: a ampliação do escopo}

O IBGE levou a campo entre agosto de $2019 \mathrm{e}$ março de 2020, a 2a edição da PNS. A ampliação do escopo foi bastante significativa. Diversas alterações foram feitas no questionário, que foi ampliado, assim como o tamanho da amostra, que atingiu ao redor de 100 mil domicílios em todo o território nacional. Por dificuldades logísticas observadas na PNS-2013, não foram realizadas medidas de parâmetros clínicos e laboratoriais.

A primeira parte do questionário ${ }^{28}$ visava a caracterizar o domićlilio. O módulo A ("informações do domicílio") trouxe questões sobre as características da habitação, fornecimento de água, esgoto, coleta de lixo, energia elétrica e presença de animais domésticos e bens de consumo. As perguntas dos módulos seguintes foram orientadas a todos os moradores do domicílio. O módulo B ("visitas domiciliares de Equipe de Saúde da Família e agentes de endemias") questionava 
sobre o registro dos moradores em unidade de saúde da família e sobre a visita de membros da eSF, agentes comunitários de saúde e de controle de endemias. O módulo C ("características gerais dos moradores") teve por objetivo coletar dados demográficos e socioeconômicos de todos os habitantes. O módulo D ("características de educação dos moradores") apresentava uma série de questionamentos sobre letramento, grau de escolaridade e frequência escolar dos moradores. Em seguida, o módulo E ("características de trabalho das pessoas 14 anos ou mais de idade"), aplicado a todos os indivíduos a partir desta faixa etária, buscava caracterizar a forma de remuneração, afastamentos do trabalho, tipo de vínculo e rendimento. Esta versão do questionário passou a incluir outras formas de trabalho, como afazeres domésticos e o cuidado de outros moradores da casa, como crianças, idosos, enfermos ou pessoas com necessidades especiais. Complementando a caracterização das pessoas, o módulo F ("rendimentos de outras fontes") investigava se a família tinha outras fontes de renda, como aposentadoria, pensão alimentícia, benefícios sociais (BPC -LOAS, Bolsa Família) etc.

Ampliado em relação ao questionário da PNS-2013, o módulo G ("pessoas com deficiências") verificava se algum dos habitantes era portador de alguma necessidade especial, qual o tipo de deficiência, o grau de limitação e se era acompanhado em algum serviço de reabilitação. O módulo I ("cobertura de plano de saúde") procurava saber se algum dos moradores tinha um plano de saúde especial ou particular, médico ou odontológico, os serviços oferecidos, os dependentes e o responsável pelo pagamento.

Com a finalidade de caracterizar o estado de saúde e a utilização de serviços de saúde pelos moradores do domicílio, o histórico módulo J ("acesso e utilização de serviços de saúde"), presente desde o suplemento da PNAD-1998, compreendia aspectos sobre limitações às atividades habituais por motivo de saúde, a descrição deste motivo, limitações causadas por problemas crônicos, tipo de estabelecimento buscado para atendimento de saúde, número de consultas médicas em 12 meses, consumação do atendimento quando buscado e qual o prestador. Havia ainda questões sobre prescrição de medicamentos, formas de acesso a eles, utilização do Programa Farmácia Popular, internações hospitalares, atendimento domiciliar de urgência, utilização de práticas integrativas e complementares etc.

O módulo K ("saúde dos indivíduos com 60 anos ou mais") captava informações sobre a auto- nomia da pessoa ao desempenhar atividades com diversos graus de dificuldade, a existência de um cuidador, uso de medicamentos, morbidades comuns da faixa etária e imunização. Já o módulo L ("crianças com menos de 2 anos de idade") abordava questões variadas sobre a saúde da criança, contemplando informações sobre alimentação, aleitamento materno, testes de rastreio neonatal e registro de dados do cartão vacinal.

Os próximos módulos integravam a segunda parte do questionário e deveriam ser respondidos apenas por um dos moradores do domicílio, com 15 anos ou mais, aleatoriamente selecionado. O módulo M ("características do trabalho e apoio social") aprofundava questões relacionadas ao trabalho, abordando, por exemplo, exposições ambientais no local. Neste módulo, também eram exploradas as relações familiares e sociais. A autopercepção do indivíduo sobre seu estado de saúde numa perspectiva ampliada foi questionada no módulo $\mathrm{N}$ ("percepção do estado de saúde"), que também indagou sobre questões específicas, como dor no peito e outras manifestações frequentes em alguns tipos de transtornos da saúde mental.

O módulo O ("acidentes") tratou da condução de automóveis e motocicletas, da adoção de medidas de segurança, da ocorrência de acidentes no trânsito e de suas consequências. Alguns hábitos de vida do indivíduo foram abordados no módulo P ("estilos de vida"), como composição da dieta, prática de atividade física, consumo de bebida alcoólica e de tabaco, modalidades de deslocamento, nível de esforço físico no trabalho, utilização de aparelhos eletrônicos etc.

Uma série de condições de saúde foram apontadas no módulo Q ("doenças crônicas"). As questões, em suma, trataram de problemas de saúde comuns. Nesta seção, o indivíduo era perguntado, por exemplo, sobre se algum médico já tinha dado algum diagnóstico de hipertensão arterial e diabetes, uso de medicamentos para tal, realização de exames, medidas para controlá -los, dentre outros aspectos relacionados a esses problemas. Outras morbidades também foram incluídas, como alterações do colesterol, doenças cardiovasculares, asma, osteoartrites, dor lombar e problemas de coluna, transtornos de saúde mental, como depressão, transtorno de ansiedade, síndrome do pânico etc.

$\mathrm{O}$ módulo R ("saúde da mulher de 15 anos ou mais de idade") destinava-se à investigação do acesso ao rastreio do câncer de colo do útero e de mama. Também englobava questões relacionadas à menstruação, menopausa, planejamento repro- 
dutivo e uso de contraceptivos. Ainda destinado apenas às mulheres, o módulo $\mathrm{S}$ ("atendimento pré-natal”) tratava da história reprodutiva, abordando questões relacionadas à rotina de acompanhamento pré-natal e ao parto.

Dedicado à saúde bucal, o módulo U trazia aspectos relacionados aos cuidados com a higiene bucal, consultas odontológicas, uso de prótese dentária etc. $\mathrm{O}$ módulo $\mathrm{Z}$ ("paternidade e prénatal do parceiro") era aplicado aos homens com 15 anos ou mais e se dedicou a coletar informações sobre filhos e acompanhamento pré-natal, por exemplo.

Na PNS-2019, as questões sobre violência foram ampliadas e reunidas no módulo V ("violência”), com sua privacidade assegurada, o próprio entrevistado podia preencher o formulário, em que era perguntado sobre situações diversas de violência, inclusive sobre o autor do ato, o local da ocorrência e suas possíveis consequências.

$\mathrm{O}$ módulo $\mathrm{T}$ (“doenças transmissíveis") questionou sobre sintomas comuns em doenças infecciosas transmissíveis, como tuberculose, doença de Chagas, hanseníase e infecções sexualmente transmissíveis.

Ao questionário da PNS-2019, também foram inseridas as questões do módulo Y ("atividade sexual"), destinadas a moradores com 18 anos ou mais. Elas exploravam a idade de iniciação sexual, hábitos relacionados ao comportamento sexual, como o uso de camisinha, e a orientação sexual dos indivíduos.

Também foi criado o módulo AA ("relações e condições de trabalho"), que continha perguntas sobre situações conflituosas no trabalho, involuntariedade, insalubridade, coerção, condições degradantes e problemas de saúde dele decorrentes. A inclusão deste módulo seguiu recomendações da Organização Internacional do Trabalho (OIT).

Em síntese, a PNS-2019 contemplou um total de 26 módulos e 803 perguntas e manteve praticamente o mesmo número de questões da PNS2013 (Quadro 2).

\section{A inclusão do Módulo de Avaliação dos Serviços de Atenção Primária na PNS-2019 a partir do Primary Care Assessment Tool (PCAT)}

A PNS-2019 dedicou, pela primeira vez, toda uma parte de seu questionário para a produção de dados sobre os serviços de APS na perspectiva do usuário adulto. O tema está contido no Módulo H ("Atendimento Médico", em substi- tuição ao módulo homônimo na PNS-2013) e foi aplicado na parte individual da entrevista aos sujeitos com 18 anos ou mais. Foram incorporadas as questões da versão reduzida do Primary Care Assessment Tool (PCAT), um dos principais instrumentos empregados na avaliação da APS.

O PCAT utiliza um questionário estruturado de perguntas fechadas para a coleta dos dados. $\mathrm{O}$ mais recente manual do instrumento, publicado pelo Ministério da Saúde ${ }^{29}$, informa a existência de inúmeras versões, conforme o público-alvo, classicamente adultos e crianças, além de adaptações para situações específicas, como a versão para profissionais de saúde (médicos, enfermeiros e cirurgiões-dentista).

O PCAT se organiza a partir da avaliação individual de cada um dos atributos da atenção primária, todos contemplados nos questionários. A cada indagação, o entrevistado deve responder com uma escala do tipo Likert em quatro pontos: "com certeza, sim", "provavelmente, sim", "provavelmente, não”, “com certeza, não”. Também existe a opção do entrevistado se abster, com a resposta "não sabe/não lembra". A cada uma das respostas é atribuído um valor de um a quatro para compor uma pontuação ("escore") através de uma média aritmética simples. Ao se basear nos atributos essenciais e derivados da APS definidos por Starfield $(2002)^{30}$ para formular suas perguntas, o objetivo primordial do PCAT é estimar o grau de extensão e orientação de um serviço para a APS, isto é, sua capacidade de "fornecer atenção integral, do ponto de vista biopsicossocial, a sua comunidade adscrita" 31 .

$\mathrm{O}$ instrumento já foi traduzido para muitos idiomas e validado em muitos países. No Brasil, sua validação e primeiras aplicações ocorreram em $2006^{32}$. Uma revisão recente identificou 42 publicações utilizando o PCAT, entre 2000 e meados de $2016^{33}$. Destes, o maior estudo em número de participantes foi realizado no Rio de Janeiro, em 2014, com 6.675 entrevistados $^{34}$. Com a PNS-2019, o país se tornou o primeiro a aplicar o instrumento em escala nacional com amostra probabilística e representativa das capitais, unidades da federação (UF) e cinco grandes regiões do país ${ }^{35}$. Isso significa que é possível calcular intervalos de confiança para as estimativas geradas pelos escores do instrumento, garantindo portanto, uma linha de base para futuros estudos de avaliação da APS em todas as UF do país.

O "PCATool-Brasil para adultos" é a versão reduzida do instrumento incorporado ao módulo H da PNS-2019. Ela foi proposta e validada por Oliveira et $\mathrm{al}^{36}$, como uma alternativa capaz 
Quadro 2. Comparação dos Módulos da Pesquisa Nacional de Saúde (PNS-2019) com a PNS-2013 e Suplementos de Saúde PNAD (1998, 2003, 2008).

\begin{tabular}{|c|c|c|c|c|c|c|}
\hline \multirow{2}{*}{\multicolumn{2}{|c|}{ Módulos na PNS-2019 }} & \multicolumn{5}{|c|}{$\mathrm{N}^{\circ}$ de questões } \\
\hline & & \multirow{2}{*}{$\begin{array}{c}\text { PNS- } \\
2019 \\
22 \\
\end{array}$} & \multirow{2}{*}{$\begin{array}{c}\text { PNS- } \\
2013 \\
24 \\
\end{array}$} & \multirow{2}{*}{$\begin{array}{c}\text { PNAD- } \\
2008 \\
0 \\
\end{array}$} & \multirow{2}{*}{$\begin{array}{c}\text { PNAD- } \\
2003 \\
0 \\
\end{array}$} & \multirow{2}{*}{\begin{tabular}{|c} 
PNAD- \\
1998 \\
0 \\
\end{tabular}} \\
\hline A & Informações do domicílio & & & & & \\
\hline B & $\begin{array}{l}\text { Visitas domiciliares de Equipes de Saúde da Família e } \\
\text { Agentes de Endemias }\end{array}$ & 4 & 4 & 0 & 0 & 0 \\
\hline $\mathrm{C}$ & Características gerais dos moradores & 16 & 12 & 0 & 0 & 0 \\
\hline $\mathrm{D}$ & Características de educação dos moradores & 18 & 15 & 0 & 0 & 0 \\
\hline $\mathrm{E}$ & $\begin{array}{l}\text { Características de trabalho das pessoas } 14 \text { anos ou mais de } \\
\text { idade }\end{array}$ & 32 & 27 & 0 & 0 & 0 \\
\hline $\mathrm{F}$ & Rendimentos de outras fontes & 9 & 10 & 0 & 0 & 0 \\
\hline G & Pessoas com deficiências & 51 & 23 & 15 & 7 & 7 \\
\hline I & Cobertura de plano de saúde & 9 & 12 & 27 & 24 & 24 \\
\hline $\mathrm{J}$ & Utilização de serviços de saúde $\left(^{*}\right)$ & 56 & 59 & 65 & 51 & 53 \\
\hline $\mathrm{K}$ & Saúde dos indivíduos com 60 anos ou mais & 37 & 60 & 0 & 0 & 0 \\
\hline $\mathrm{L}$ & Crianças com menos de 2 anos de idade & 24 & 20 & 0 & 0 & 0 \\
\hline $\mathrm{M}$ & Características do trabalho e apoio social & 21 & 20 & 0 & 0 & 0 \\
\hline $\mathrm{N}$ & Percepção do estado de saúde & 16 & 22 & 0 & 0 & 0 \\
\hline $\mathrm{O}$ & Acidentes & 22 & 48 & 8 & 0 & 0 \\
\hline $\mathrm{P}$ & Estilos de vida $\left(^{* *}\right)$ & 90 & 85 & 108 & 0 & 0 \\
\hline Q & Doenças crônicas & 141 & 136 & 0 & 0 & 0 \\
\hline $\mathrm{R}$ & $\begin{array}{l}\text { Saúde da Mulher (mulheres de } 15 \text { anos ou mais de idade) } \\
(* * *)\end{array}$ & 35 & 49 & 16 & 3 & 0 \\
\hline S & Atendimento pré-natal & 63 & 64 & 0 & 0 & 0 \\
\hline $\mathrm{U}$ & Saúde bucal & 13 & 19 & 0 & 0 & 0 \\
\hline Z & $\begin{array}{l}\text { Paternidade e pré-natal do parceiro (homens de } 15 \text { anos ou } \\
\text { mais) }\end{array}$ & 14 & 0 & 3 & 0 & 0 \\
\hline $\mathrm{V}$ & Violência (pessoas com 18 anos ou mais) & 21 & 0 & 0 & 0 & 0 \\
\hline $\mathrm{T}$ & Doenças transmissíveis & 6 & 0 & 0 & 0 & 0 \\
\hline Y & Atividade sexual (18 anos ou mais) & 8 & 0 & 0 & 0 & 0 \\
\hline AA & Relações e condições de trabalho (18 anos ou mais) & 43 & 0 & 0 & 0 & 0 \\
\hline $\mathrm{H}$ & Atendimento médico (18 anos ou mais) & 30 & 30 & 0 & 0 & 0 \\
\hline $\mathrm{W}$ & Antropometria (15 anos ou mais de idade) & 2 & 12 & 0 & 0 & 0 \\
\hline & Total: & 803 & 751 & 242 & 85 & 84 \\
\hline
\end{tabular}

Nota: Os módulos destacados em cinza são aqueles que a partir da PNS-2013 passaram a ter apenas um morador de cada domicílio selecionado para responder ao módulo, ao contrário dos demais módulos em que todos os moradores do mesmo domicílio respondem.

(*) Nas PNAD 1998, 2003 e 2008, esse módulo continha as partes sobre "morbidade", "acesso aos serviços de saúde", "internação" e "atendimento de urgência no domicílio". (**) Na PNAD-2008, foram aqui agrupados os módulos de sedentarismo, tabagismo - questões gerais e pesquisa especial de tabagismo para pessoas com 15 anos ou mais. ${ }^{* * *}$ ) Nas PNAD 2003 e 2008, o escopo era menor e o módulo se chamava "Acesso aos serviços preventivos de saúde de mulheres de 25 anos ou mais".

Fonte: Elaboração dos autores.

de otimizar o processo de coleta e de utilização dos resultados, adequando-se ao já extenso questionário da PNS-201937.

Como dito, o questionário do PCAT, mesmo em sua versão reduzida, é dividido entre os atributos da APS. Todas as perguntas se relacionam ao último médico que atendeu o entrevistado em uma unidade básica de saúde. A avaliação do "acesso - primeiro contato" é feita por questiona- mentos sobre a identificação deste médico como primeira referência quando ocorre algum novo problema de saúde, sobre acessibilidade e agenda. A "longitudinalidade" está dividida em quatro questões e a "coordenação do cuidado" em seis, tratando de encaminhamento para atenção secundária e sistemas de informações. Na seção sobre "integralidade" questiona-se sobre os serviços oferecidos e disponíveis na unidade de APS. 
Há ainda mais duas seções sobre “orientação familiar" e "orientação comunitária”.

\section{Considerações finais}

Inquéritos populacionais têm sido desenvolvidos em todo o mundo, em diferentes contextos e modelos, ocupando uma posição de relevância na produção de informação em saúde. Ao longo do tempo, foram apontadas limitações e apresentadas observações sobre o método. Por exemplo, a coleta de dados sobre a presença de morbidades a partir do autorrelato do entrevistado ("morbidade referida") se baseia na percepção pessoal do sujeito, um dado controverso para muitos pesquisadores, que enfatizam a necessidade de se desenvolver e validar instrumentos que permitam que essa medida seja mais confiável ${ }^{38}$. A crescente "taxa de não-resposta" nos inquéritos domiciliares tem precipitado uma discussão sobre possíveis prejuízos à qualidade dos resultados. Acredita-se que o desinteresse dos indivíduos tenha relação com o aumento do número de pesquisas e a maior preocupação com a privacidade de dados. Nos países em desenvolvimento, em contrapartida, a adesão parece bem maior ${ }^{39}$.

Garantir a periodicidade dos inquéritos é essencial para que os resultados possam ser acompanhados evolutivamente. Alguns países, como a Nova Zelândia ${ }^{40}$, têm desenvolvido pesquisas domiciliares de saúde contínuas, fornecendo, assim, informações em períodos muito mais curtos. Por outro lado, a execução dos inquéritos domiciliares exige altos investimentos financeiros. A fim de maximizar o aproveitamento desses recursos, há autores que desencorajam a realização de inquéritos com espectros restritos, como os específicos para uma só doença, realizados em alguns países em desenvolvimento. Eles defendem que com um pequeno acréscimo de verba, poderiam ampliar a sua abordagem e fornecer mais informações sobre a saúde da população ${ }^{41}$.

Recomenda-se que os diversos inquéritos domiciliares procurem harmonizar os seus desenhos, de modo a garantir a comparabilidade dos dados obtidos. A divulgação dos microdados dessas pesquisas, a linkage e anonimização dos mesmos com outras bases de dados, assim como o desenvolvimento de entrevistas de abrangência intersetorial, também são encorajados ${ }^{42,43}$. A incorporação do PCAT no questionário da PNS2019, pelo IBGE, avança neste sentido, produzindo dados relevantes sobre os serviços de APS e que poderão ser comparados com os resultados de dezenas de estudos que utilizam a mesma ferramenta, no Brasil e no mundo. Esta medida é um reconhecimento da utilidade e importância do PCAT na avaliação deste nível de cuidado.

No contexto contemporâneo de obtenção de dados em grande volume, velocidade e variedade (Big Data), a produção de informações comparáveis parece ainda mais relevante. Atualmente, dados de navegação em redes sociais, informações pessoais de usuários, registros de dispositivos vestíveis, por exemplo, têm sido utilizados como fontes de dados. Na saúde, há ainda as informações dos registros médicos em bases eletrônicas. Apesar dos inúmeros questionamentos quanto ao uso dessas informações, com destaque para a discussão sobre privacidade, o Big Data tem sido apresentado como uma ferramenta promissora na obtenção de informação em saúde no futuro ${ }^{44-46}$.

Em resposta à demanda por informações em tempo real no âmbito da pandemia de COVID-19, em 2020, o IBGE conseguiu, em menos de três meses, planejar, elaborar o instrumento, capacitar à distância os entrevistadores, aplicar o questionário, criticar a base de dados, tabular e divulgar, em meados de junho de 2020, a "PNAD COVID-19”, uma versão especial do inquérito dedicada a recolher informações durante o período da pandemia. As entrevistas foram realizadas por telefone, alcançando cerca de 193 mil domicílios, por mês, em todo o país. O seu questionário abrangeu características sociodemográficas, de trabalho e renda, além de investigar a presença de sintomas de síndrome gripal e, dentre esses, aqueles mais associados à COVID-19, além de coletar informações sobre a procura por estabelecimentos de saúde, com ou sem internação, pelas pessoas sintomáticas. ${ }^{47} \mathrm{O}$ IBGE inovou e pela primeira vez em sua história de quase 90 anos, realizou coleta de dados de base domiciliar com suporte telefônico e transmissão em tempo real dos dados coletados. Em tempos de crise sanitária esse será um grande aprendizado e legado para futuras pesquisas de base populacional do Instituto. 


\section{Colaboradores}

VSTM Silva estruturou o artigo de revisão. LF Pinto revisou o texto criticamente e validou a versão final.

\section{Referências}

1. Ross DA, Vaughan JP. Health interview surveys in developing countries: a methodological review. Stud Fam Plann 1986; 17(2):78-94.

2. Barros $\mathrm{MB}$ de $\mathrm{A}$. Inquéritos domiciliares de saúde: potencialidades e desafios. Rev Bras Epidemiol 2008; 11(Suppl. 1):6-19.

3. Szwarcwald CL, Damacena GN. Amostras complexas em inquéritos populacionais: planejamento e implicações na análise estatística dos dados. Rev Bras Epidemiol 2008; 11(Supl. 1):38-45.

4. Bruin A, Picavet HSJ, Nossikov A, organizadores. Health interview surveys: towards international harmonization of methods and instruments. Copenhagen: World Health Organization, Regional Office for Europe; 1996.161 p. (WHO regional publications).

5. Viacava F. Informações em saúde: a importância dos inquéritos populacionais. Cien Saude Colet 2002; 7(4):607-621.

6. Béland Y. Canadian community health survey--methodological overview. Health Rep 2002; 13(3):9-14.

7. European Commission. Eurostat. European Health Interview Survey (EHIS wave 3). Methodological manual: 2020 edition (re-edition). Luxemburgo: Publications Office of the European Union; 2020

8. Instituto Nacional de Estatística (INE). Inquérito Nacional de Saúde: 2014. Lisboa: INE; 2016. [acesado 2020 Jul 15]. Disponível em: https://www.ine.pt/xurl/ pub/263714091.

9. Gobierno de España. Ministerio de Sanidad, Consumo y Bienestar Social. Encuesta Nacional de Salud de España 2017. [acesado 2020 Jul 15]. Disponível en: https://www.mscbs.gob.es/estadEstudios/estadisticas/ encuestaNacional/encuesta2017.ht.

10. Istituto Nazionale di Statistica. Indagine Multiscopo Sulle Famiglie: Aspetti Della Vita Quotidiana. 2020. [acessado 2020 Jul 15]. Disponível em https://www. istat.it/it/archivio/91926

11. He H, Pan L, Pa L, Cui Z, Ren X, Wang D, Liu F, Wang X, Du J, Wang H, Wan S, Zhao J, Peng X, Wang X, Zhang J, Wang Y, Ren H, Yu C, Shan G. Data Resource Profile: The China National Health Survey (CNHS). Int J Epidemiol 2018; 47(6):1734-1735.

12. International Institute for Population Sciences (IIPS) and ICF. National Family Health Survey (NFHS-4), 2015-16: India. Mumbai: IIPS; 2017.

13. Australian Bureau of Statistics. National Health Survey: First Results, 2017-18. Canberra: ABS; 2018.

14. Jatrana S, Crampton P. Continuity of care with general practitioner in New Zealand: results from SoFIE-Primary Care. New Zealand Med J. 2011; 124 (1329):16-25.

15. Brasil. Instituto Brasileiro de Geografia e Estatística (IBGE). Diretoria de Pesquisas. Estatísticas da saúde: assistência médico-sanitária 2009. Rio de Janeiro: IBGE; 2010.

16. Oliveira LAP, Simões CCS. O IBGE e as pesquisas populacionais. Rev Bras Estud Popul 2005; 22(2):291302. 
17. Pinto LF, Freitas MPS, Figueiredo AWS. Sistemas Nacionais de Informação e levantamentos populacionais: algumas contribuições do Ministério da Saúde e do IBGE para a análise das capitais brasileiras nos últimos 30 anos. Cien Saude Colet 2018; 23(6):18591870.

18. Brasil. Instituto Brasileiro de Geografia e Estatística (IBGE). Diretoria de Pesquisas. Coordenação de Trabalho e Rendimento. Pesquisa Nacional por Amostra de Domicílios Contínua. Notas técnicas. Versão 1.6. Rio de Janeiro: IBGE; 2019.

19. Travassos C, Viacava F, Laguardia J. Os Suplementos Saúde na Pesquisa Nacional por Amostra de Domicílios (PNAD) no Brasil. Rev Bras Epidemiol 2008; 11(Supl. 1):98-112.

20. Brasil. Instituto Brasileiro de Geografia e Estatística (IBGE). Diretoria de Pesquisas. Coordenação de Trabalho e Rendimento. Pesquisa Nacional por Amostra de Domicílios. Acesso e utilização de serviços de saúde 2003. Rio de Janeiro: IBGE; 2005.

21. Brasil. Instituto Brasileiro de Geografia e Estatística (IBGE). Diretoria de Pesquisas. Coordenação de Trabalho e Rendimento. Pesquisa Nacional por Amostra de Domicílios. Um panorama da saúde no Brasil. Acesso e utilização dos serviços, condições de saúde e fatores de risco e proteção à saúde 2008. Rio de Janeiro: IBGE; 2010.

22. Souza-Júnior PRB, Freitas MPS, Antonaci GA, Szwarcwald CL. Desenho da amostra da Pesquisa Nacional de Saúde 2013. Epidemiol Serv Saude 2015; 24(2):207216.

23. Malta DC, Szwarcwald CL, Silva Júnior JB. Primeiros resultados da análise do laboratório da Pesquisa $\mathrm{Na}$ cional de Saúde. Rev bras epidemiol. 2019; 22(Suppl. 2):E190001.SUPL.2.

24. Szwarcwald CL, Malta DC, Pereira CA, Vieira MLFP, Conde WL, Souza Júnior PRB de, et al. Pesquisa Nacional de Saúde no Brasil: concepção e metodologia de aplicação. Cien Saude Colet 2014; 19(2):333-342.

25. Brasil. Instituto Brasileiro de Geografia e Estatística (IBGE). Diretoria de Pesquisas. Coordenação de Trabalho e Rendimento. Pesquisa Nacional de Saúde 2013. Manual de entrevista. Rio de Janeiro: IBGE; 2013.

26. Brasil. Instituto Brasileiro de Geografia e Estatística (IBGE). Diretoria de Pesquisas. Coordenação de Trabalho e Rendimento. Pesquisa Nacional de Saúde 2013. Ciclos de Vida. Rio de Janeiro: IBGE; 2015.

27. Instituto Brasileiro de Geografia e Estatística (IBGE). Diretoria de Pesquisas. Coordenação de Trabalho e Rendimento. Pesquisa Nacional de Saúde 2013. Acesso e utilização dos serviços de saúde. Rio de Janeiro: IBGE; 2015.

28. Instituto Brasileiro de Geografia e Estatística (IBGE). Diretoria de Pesquisas. Coordenação de Trabalho e Rendimento. Pesquisa Nacional de Saúde 2019. Questionário dos moradores do domicílio. Rio de Janeiro: IBGE; 2019.
29. Brasil. Ministério da Saúde (MS). Secretaria de Atenção Primária à Saúde. Departamento de Saúde da Família. Manual do Instrumento de Avaliação da Atenção Primária à Saúde: PCATool-Brasil-2020. Ministério da Saúde, Secretaria de Vigilância em Saúde, Departamento de Vigilância Epidemiológica. Brasília: MS; 2020.

30. Starfield B. Atenção primária: equilíbrio entre necessidades de saúde, serviços e tecnologia. Brasília: Unesco, Ministério da Saúde; 2002.

31. Brasil. Ministério da Saúde (MS). Secretaria de Atenção em Saúde. Departamento de Atenção Básica. Manual do instrumento de avaliação da atenção primária à saúde: primary care assessment tool pcatool - Brasil. Brasília: MS; 2010.

32. Harzheim E, Duncan BB, Stein AT, Cunha CR, Goncalves MR, Trindade TG, Oliveira MMC, Pinto MEB. Quality and effectiveness of different approaches to primary care delivery in Brazil. BMC Health Serv Res. 2006; 6(1):156.

33. D'Avila OP, Pinto LFS, Hauser L, Gonçalves MR, Harzheim E. O uso do Primary Care Assessment Tool (PCAT): uma revisão integrativa e proposta de atualização. Cien Saude Colet 2017; 22(3):855-865.

34. Harzheim E, Hauser L, Pinto LF. Avaliação do grau de orientação para Atenção Primária em Saúde: a experiência dos usuários das Clínicas da Família e Centros Municipais de Saúde na cidade do Rio de Janeiro. Porto Alegre: UFRGS; 2015. (Relatório Final da Pesquisa PCATool -Rio-2014).

35. Harzheim E, Felipe Pinto L, D’Avila OP, Hauser L. Following the legacy of professors Barbara Starfield and Leiyu Shi in Brazil as health policy: the National Health Survey (PNS), led by the Brazilian National Institute of Geography and Statistics (IBGE) and the Primary Care Assessment Tool (PCAT). Int J Equity Health 2019; 18(1):176.

36. Oliveira MMC, Harzheim E, Riboldi J, Duncan BB. PCATool-ADULTO-BRASIL: uma versão reduzida. Rev Bras Med Fam Comunidade 2013; 8(29):256-263.

37. Brasil. Instituto Brasileiro de Geografia e Estatística (IBGE). Pesquisa Nacional de Saúde (PNS-2019): instrumento de coleta. Rio de Janeiro, 2020. [acessado 2020 jul 16]. Disponível em: https://biblioteca. ibge.gov.br/visualizacao/instrumentos_de_coleta/ doc5569.pdf.

38. Mathers CD, Murray CJ, Ezzati M, Gakidou E, Salomon JA, Stein C. Population health metrics: crucial inputs to the development of evidence for health policy. Popul Health Metrics 2003; 1(1):6

39. Meyer BD, Mok WKC, Sullivan JX. Household Surveys in Crisis. J Econ Perspectives 2015; 29(4):199-226.

40. Clark RG, Templeton R, McNicholas A. Developing the design of a continuous national health survey for New Zealand. Popul Health Metrics 2013; 11(1):25.

41. Boerma JT, Stansfield SK. Health statistics now: are we making the right investments? Lancet 2007; 369(9563):779-786. 
42. Dandona R, Pandey A, Dandona L. A review of national health surveys in India. Bull World Health Organ 2016; 94(4):286-296A.

43. Macfarlane SB. Harmonizing health information systems with information systems in other social and economic sectors. Bull World Health Organ 2005; 83(8):590-596.

44. Hansen MM, Miron-Shatz T, Lau AYS, Paton C. Big Data in Science and Healthcare: A Review of Recent Literature and Perspectives: Contribution of the IMIA Social Media Working Group. Yearb Med Inform 2014; 23(01):21-26.

45. Japec L, Kreuter F, Berg M, Biemer P, Decker P, Lampe C, Lane J, O’Neil C, Usher A. Big Data in Survey Research: AAPOR Task Force Report. PUBOPQ 2015; 79(4):839-880.

46. Wyber R, Vaillancourt S, Perry W, Mannava P, Folaranmi T, Celi LA. Big data in global health: improving health in low- and middle-income countries. Bull World Health Organ 2015; 93(3):203-208.

47. Brasil. Instituto Brasileiro de Geografia e Estatística (IBGE). Pesquisa Nacional por Amostra de Domicílios Contínua (PNAD) COVID-19: instrumento de coleta. Rio de Janeiro, 2020. [acessado 2020 jul 15]. Disponível em: https://biblioteca.ibge.gov.br/visualizacao/instrumentos_de_coleta/doc5586.pdf.

Artigo apresentado em 01/08/2020

Aprovado em 10/09/2020

Versão final apresentada em 12/09/2020

Editores-chefes: Maria Cecília de Souza Minayo, Romeu

Gomes, Antônio Augusto Moura da Silva 